\title{
Comparative evaluation of FAST or SLOW crystalloid resuscitation on oxygen delivery in a human model of haemorrhagic shock
}

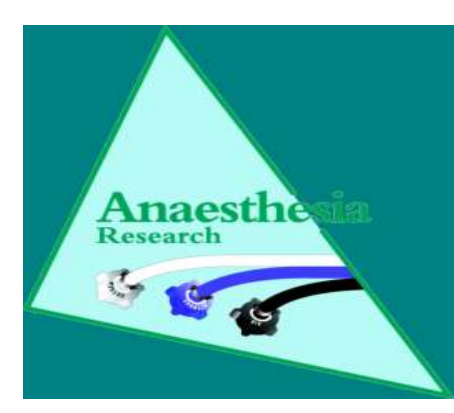

\author{
Weinberg L'1, Ho L', Lau L², Reidel B ${ }^{3}$, Churilov L4, Hahn R $^{5}$ \\ 1 Department of Anaesthesia \& Surgery, Austin Hospital, University of Melbourne, Victoria, Australia \\ 2 Department of Surgery, Austin Hospital, Victoria, Australia \\ ${ }^{3}$ Department of Anaesthesia, Peter MacCallum Cancer Centre, Victoria, Australia \\ ${ }^{4}$ The Florey Institute of Neuroscience \& Mental Health, Australia \\ ${ }^{5}$ Department of Anaesthesia, Södertälje Hospital, Södertälje, Sweden
}

Email: laurence.weinberg@austin.org.au

\section{Background and Goals:}

The current Advanced Trauma Life Support (ATLS) guidelines for the management of haemorrhagic shock advocate rapid infusion of 1-2L of crystalloid solution in the absence of the matched blood products. This traditional teaching of resuscitation appears to be based on expert opinion with minimal supporting evidence from human clinical trials. Furthermore, there is no current consensus regarding exactly what defines a "fluid bolus" and the most effective rate of fluid resuscitation in haemorrhagic shock is unknown. Although animal studies have shown that resuscitation with a fast fluid infusion had worse outcome compared to a slow fluid infusion in various models of haemorrhagic shoc, prospective research in humans on the same comparison is lacking. While fast crystalloid resuscitation has been associated with increased mortality and complications in patients with severe sepsis, there are no human studies to date comparing the rate of crystalloid resuscitation for patients in haemorrhagic shock. STUDY AIMS: Therefore we evaluated, in a model of compensated haemorrhagic shock, whether a slow (SLOW treatment) or fast (FAST treatment) rate of resuscitation with crystalloid infusion would differ in terms of oxygen delivery $\left(\mathrm{DO}_{2}\right)$, pressureand flow-based haemodynamic variables, and clinical biochemistry including $\mathrm{N}$ terminal pro B-type natriuretic peptide (NT-BNP). The STUDY DESIGN is summarised in Figure 1.

\section{Results:}

Nine normotensive healthy adult volunteers participated. No significant differences were observed in $\mathrm{DO}_{2}$ and biochemical variables between the SLOW and FAST groups. Blood volume was reduced by $16 \%$ following venesection, with a corresponding $5 \%$ reduction in cardiac index $(p<0.001)$. Immediately following resuscitation the increase in blood volume corresponded to $54 \%$ of the infused volume under FAST treatment and $69 \%$ of the infused volume under SLOW treatment $(p=0.03)$. This blood volume expansion attenuated with time to $24 \%$ and $25 \%$ of the infused volume 30 minutes post-infusion (Figure 2). During fluid resuscitation, blood pressure was higher under FAST treatment. However, cardiac index paradoxically decreased in most participants under FAST treatment during the resuscitation phase; a finding not observed under SLOW treatment (Figure 3).

\section{Clinical implications:}

Our findings contradict current resuscitation practices, which are based on presumption that FAST crystalloid resuscitation is superior to SLOW crystalloid resuscitation. There were no significance differences in $\mathrm{DO}_{2}$ between the FAST and SLOW treatments groups, and changes in cardiac index and blood pressure did not reflect the magnitude of intravascular blood volume deficit. Crystalloid resuscitation expanded intravascular blood volume by approximately $25 \%$. This was associated with a reduction in cardiac index and stroke volume index during the resuscitation phase, again supporting the theory of over resuscitation and suboptimal myocardial performance. In the view of the increasing prevalence of cardiovascular disease with advancing age, the impact of FAST resuscitation on the cardiac index observed in this study, which is of most relevant to combat casualties, might be more profound in the current aging population or patients with pre-existing cardiac dysfunction. Older subjects have a more poorly functioning adrenergic system, although stress hormones are generally higher than in younger subjects, which makes them more sensitive to changes in preloading and more prone to hypotension in hypovolaemia. Finally, the discrepancy between changes in blood volume and cardiac index could mislead the clinician who tries to maintain the blood volume by monitoring cardiac index alone. Neither did the reduction in cardiac index proportionally reflect the degree of blood loss from the venesection, nor did the increase in cardiac index accurately reflect the persisting hypovolaemic state.

\section{Conclusion:}

In a healthy volunteer model of compensated haemorrhagic shock, FAST or SLOW fluid resuscitation had no significant impact on $\mathrm{DO}_{2}$ between treatments groups. In both groups, changes in cardiac index and blood pressure did not reflect the magnitude of intravascular blood volume deficit. Crystalloid resuscitation expanded intravascular blood volume by approximately $25 \%$.

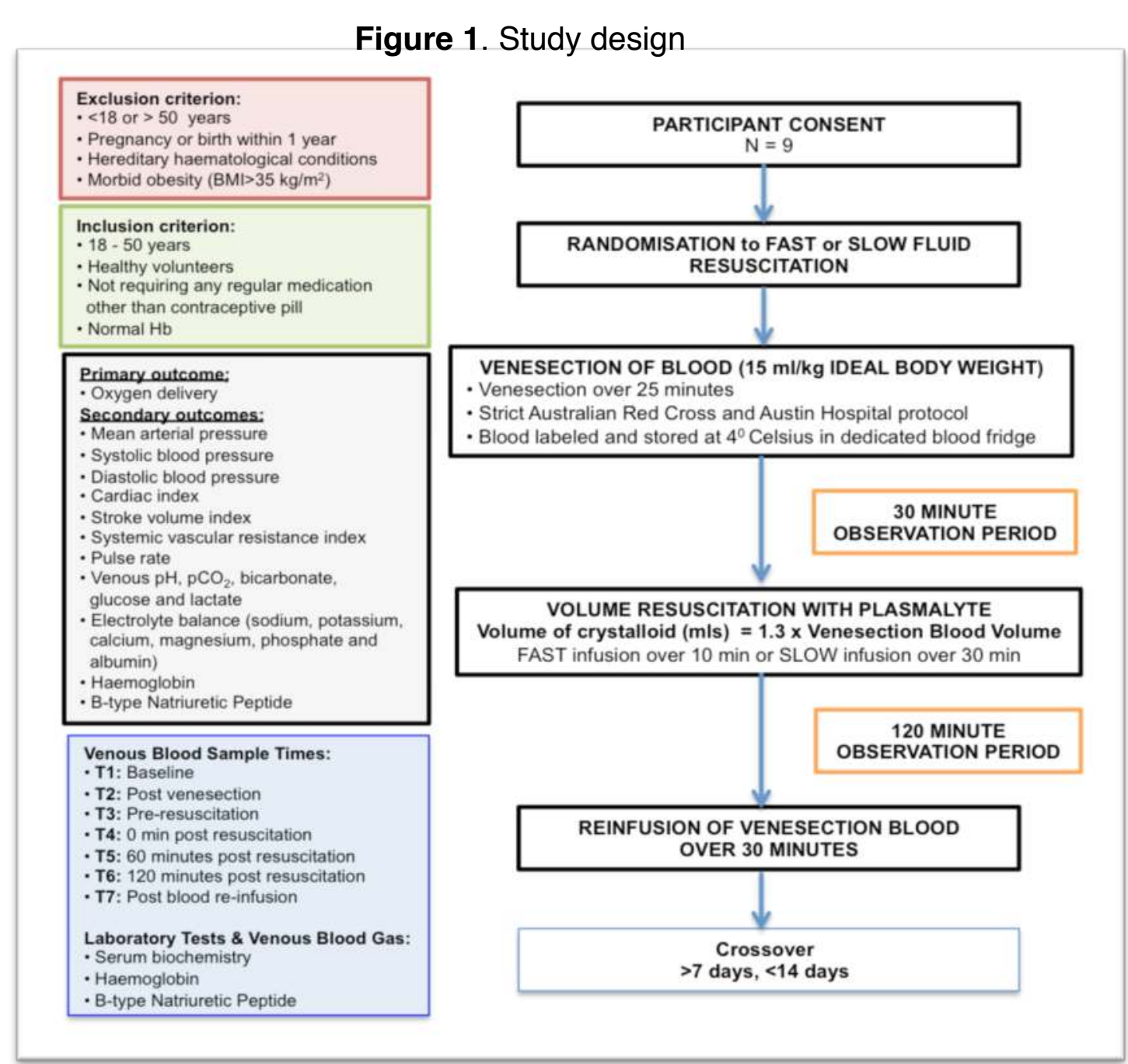

Figure 2. Changes in percentage in cardiac index in relation to blood volume in a human model of compensated haemorrhagic shock. (T1=Baseline; T2=Post-Venesection; T3=PreResuscitation; T4=0-min Post-Resuscitation; T5=60-min Post-Resuscitation; T6=120-min Post Resuscitation; T7=Post Blood Transfusion)

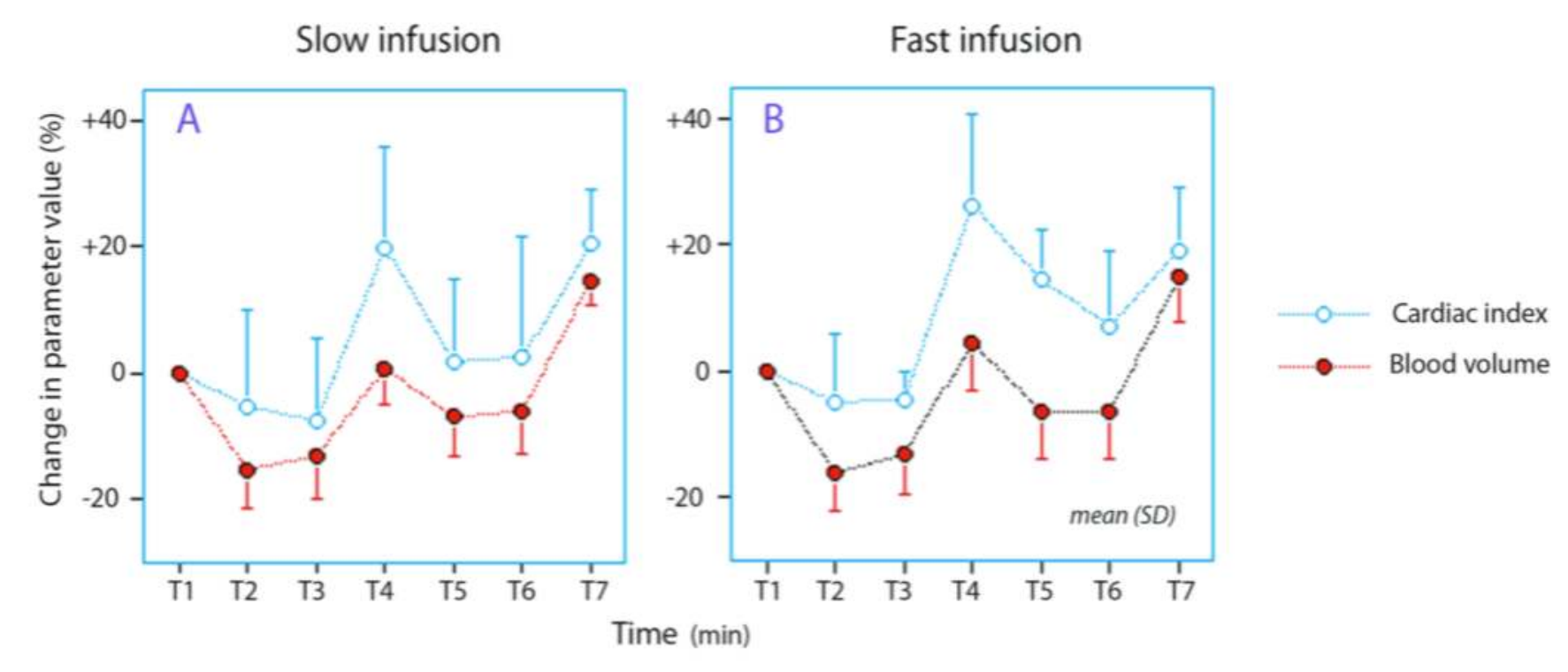

Figure 3. Box-and-Whisker plot showing median changes in cardiac index observed during FAST (20mL.kg-1 crystalloid over 10 minutes) and SLOW (20mL.kg-1 crystalloid over 30 minutes) resuscitation in a human model of compensated haemorrhagic shock.

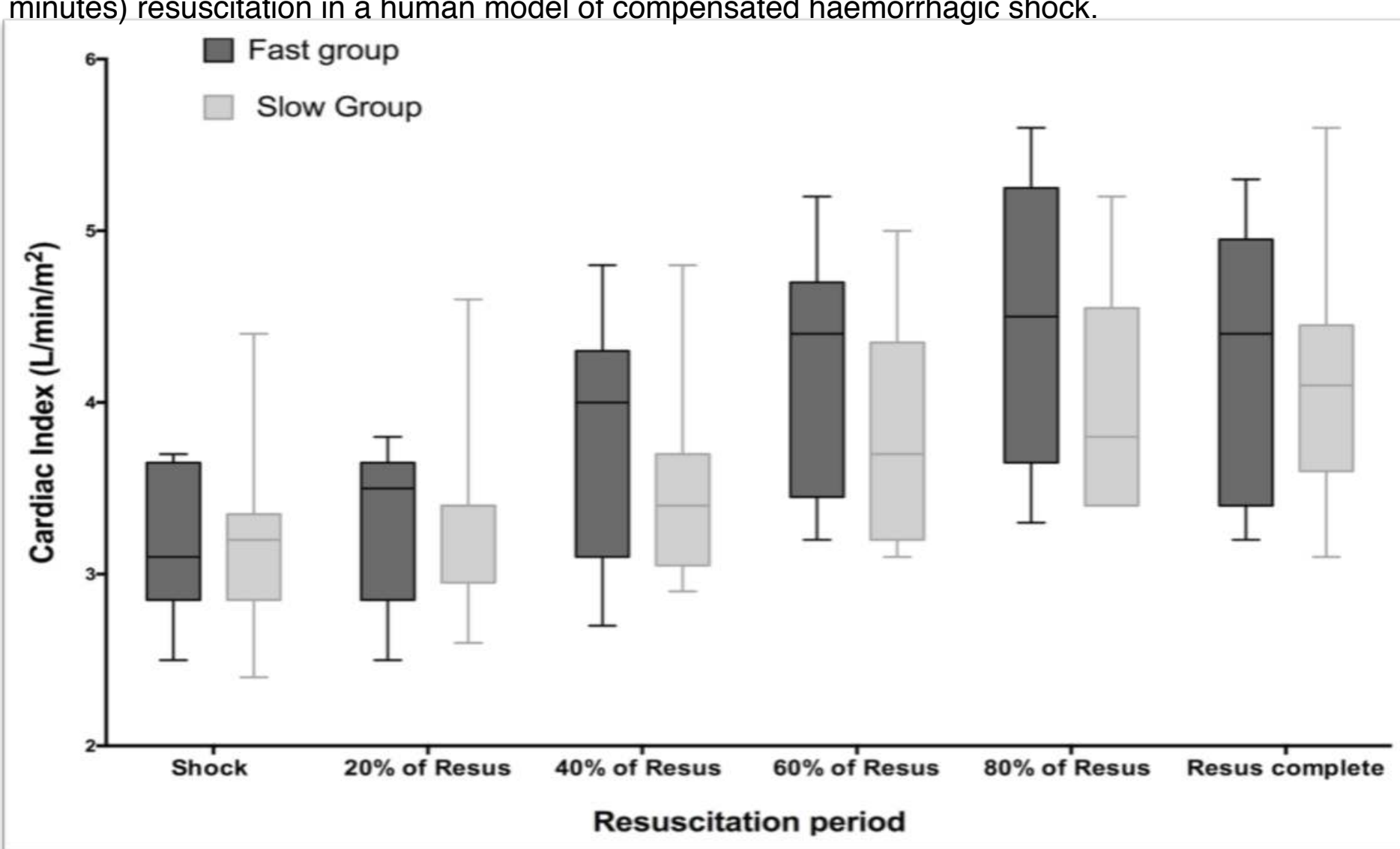

